Proyecciones Journal of Mathematics

Vol. 36, No 3, pp. 499-510, September 2017.

Universidad Católica del Norte

Antofagasta - Chile

\title{
Six dimensional matrix summability of triple sequences
}

\author{
Bimal Chandra Das \\ Tripura University, India \\ Received : January 2017. Accepted : January 2017
}

\begin{abstract}
In this paper we introduced the $R H$-regularity condition of six dimensional matrix. Matrix summability is one of the important tool used to characterize sequence spaces. In 2004 Patterson presented such a characterization of bounded double sequence using four dimensional matrix. Our main aim is to extend Patterson result in triple sequence spaces using six dimensional matrix transformations.
\end{abstract}

Key Words: Triple sequence, $R H$-regular, regular matrix transformation.

AMS Classification: 40A05, 40B05, 40A99. 


\section{Introduction and Preliminaries}

Throughout the article a triple sequence $x$ is denoted by $\left(x_{p, q, r}\right)$ i.e. a triple infinite array of real or complex numbers $x_{p, q, r} ; p ; q ; r \in N$. Throughout $\mathrm{N}$, $\mathrm{R}$ and $\mathrm{C}$ denote the set of natural, real and complex numbers respectively.

At the initial stage different types of notions of triple sequences were introduced and investigated by Sahiner, Gurdal and Duden [3]. Sahiner and Tripathy [4] studied I-related properties in triple sequence spaces. Debnath, Sharma and Das [15] and Debnath and Das [16] generalized these concepts by using the difference operator. Recently Debnath, Das, Bhattacharya and Debnath [17] studied regular matrix transformation on triple sequence spaces and showed some interesting results. Triple sequences have also been studied by Tripathy and Goswami ([5],[6],[7],[8]) and many others.

The Silverman-Toeplitz [13] theorem characterizes the regularity of two dimensional matrix transformation. Robison [10] has given definitions for giving a value to a divergent double series by considering the double sequence for the series and established the conditions of regularity of linear transformations on double sequence spaces. Since then this concept has been studied by many researchers. In 2004 Patterson [14] presented an accessible multidimensional analog of theorem of Brudno [1] using four dimensional matrix transformation of double sequences. In this paper the aim of the author is to extend results of Patterson in triple sequence spaces using six dimensional matrix transformations. In addition, we introduced the $R H$-regularity condition of six dimensional matrix.

Definition 1.1. A triple sequence $\left(x_{p, q, r}\right)$ is said to be convergent to $L$ in Pringsheim's sense (denoted by $P-\lim x=\mathrm{L}$ ) if for every $\epsilon>0$, there exists $n_{0} \in N$ such that

$$
\left|x_{p, q, r}-L\right|<\epsilon, \text { whenever } p \geq n_{0}, q \geq n_{0}, r \geq n_{0},
$$

and we write

$$
\lim _{p, q, r \rightarrow \infty} x_{p, q, r}=L .
$$

Note: A triple sequence convergent in Pringsheim's sense is not necessarily bounded [3].

Definition 1.2. A triple sequence $\left(x_{p, q, r}\right)$ is called definite divergent, if 
for every (arbitrarily large) $G>0$ there exist $n_{1}, n_{2}, n_{3} \in N$ such that $\left|x_{p, q, r}\right|>G$ for $p \geq n_{1}, q \geq n_{2}, r \geq n_{3}$.

Definition 1.3. A triple sequence $\left(x_{p, q, r}\right)$ is divergent in the Pringsheim sense $\left(P\right.$-divergent) provided that $\left(x_{p, q, r}\right)$ does not converge in the Pringshiem sense ( $P$-convergent).

Definition 1.4. A triple sequence $\left(x_{p, q, r}\right)$ is said to be bounded if there exist $M>0$ such that $\left|x_{p, q, r}\right|<M$ for all $p, q, r \in N$.

Definition 1.5. Let $A$ denote a six dimensional summability method that maps the complex triple sequence $x$ into the triple sequence $A x$ where the $l m n$-th term to $A x$ is as follows:

$(A x)_{l, m, n}=\sum_{p=1}^{\infty} \sum_{q=1}^{\infty} \sum_{r=1}^{\infty} a_{l, m, n, p, q, r} x_{p, q, r}$

Definition 1.6. The six dimensional matrix $A$ is said to be $R H$-regular if it maps every bounded $P$-covergent sequence into a $P$ - convergent sequence with the same $P$-limit.

Theorem 1.1. ([11],[10]) The four dimensional matrix $A$ is $R H$-regular if and only if

$R H_{1}: P-\lim _{m, n \rightarrow \infty} a_{m, n, p, q}=0$, for each $p$ and $q$;

$R H_{2}: P-\lim _{l, m \rightarrow \infty} \Sigma_{p=1, q=1}^{\infty, \infty} a_{l, m, p, q}=1 ;$

$R H_{3}: P-\lim _{l, m \rightarrow \infty} \Sigma_{p=1}^{\infty}\left|a_{l, m, p, q}\right|=0$, for each $q ;$

$R H_{4}: P-\lim _{l, m \rightarrow \infty} \Sigma_{q=1}^{\infty}\left|a_{l, m, p, q}\right|=0$, for each $p ;$

$R H_{5}: \Sigma_{p=1, q=1}^{\infty, \infty}\left|a_{l, m, p, q}\right|$ is $P$ - convergent; and

$R H_{6}$ : there exist finit positive integers $A$ and $B$ such that $\Sigma_{p, q>B} \mid$ $a_{l, m, p, q} \mid<A$

we introduce the regularity condition of six dimensional matrix as follows:

Theorem 1.2. The six dimensional matrix $A$ is $R H$-regular if and only if 
$R H_{1}: P-\lim _{l, m, n \rightarrow \infty} a_{l, m, n, p, q, r}=0$, for each $p, q$ and $r$;

$R H_{2}: P-\lim _{l, m, n \rightarrow \infty} \Sigma_{p=1, q=1, r=1}^{\infty, \infty, \infty} a_{l, m, n, p, q, r}=1 ;$

$R H_{3}: P-\lim _{l, m, n \rightarrow \infty} \Sigma_{p=1}^{\infty}\left|a_{l, m, n, p, q, r}\right|=0$, for each $q$ and $r$;

$R H_{4}: P-\lim _{l, m, n \rightarrow \infty} \Sigma_{q=1}^{\infty}\left|a_{l, m, n, p, q, r}\right|=0$, for each $p$ and $r$;

$R H_{5}: P-\lim _{l, m, n \rightarrow \infty} \Sigma_{r=1}^{\infty}\left|a_{l, m, n, p, q, r}\right|=0$, for each $p$ and $q$;

$R H_{6}: \Sigma_{p=1, q=1, r=1}^{\infty, \infty, \infty}\left|a_{l, m, n, p, q, r}\right|$ is $P$ - convergent;

$R H_{7}$ : there exist finit positive integers $A$ and $B$ such that $\Sigma_{p, q, r>B} \mid$ $a_{l, m, n, p, q, r} \mid<A$;

\section{Main Results}

Theorem 2.1. If $A=\left(a_{l, m, n, p, q, r}\right)$ and $B=\left(b_{l, m, n, p, q, r}\right)$ be two six dimensional $R H$-regular summability matrices sum a bounded triple sequence $\left(x_{p, q, r}\right)$ to the same sum, then there exist a bounded triple sequence which is summed by $B$ as by $A$.

Proof. We consider a sequence $\left(x_{l, m, n}\right)$ such that

$P-\lim _{l, m, n}(A x)_{l, m, n}=0$ and $P-\lim _{l, m, n}(B x)_{l, m, n}=0$

Let

$\chi_{l, m, n}=\Sigma_{p=1, q=1, r=1}^{\infty, \infty, \infty} a_{l, m, n, p, q, r} x_{p, q, r}$ and $\psi_{l, m, n}=\Sigma_{p=1, q=1, r=1}^{\infty, \infty, \infty} b_{l, m, n, p, q, r} x_{p, q, r}$

Since $A=\left(a_{l, m, n, p, q, r}\right)$ and $B=\left(b_{l, m, n, p, q, r}\right)$ be two six dimensional $R H$-regular summability matrices and both are summed to 0 , then there exist $\epsilon_{l, m, n}>0$ with Pringshiem limit zero such that

$\left|\chi_{l, m, n}\right| \leq \epsilon_{l, m, n}$ and $\left|\psi_{l, m, n}\right| \leq \epsilon_{l, m, n}$

Let $U:=\max _{p, q, r}\left|x_{p, q, r}\right|$ and $V:=\max _{p, q, r}\left|a_{l, m, n, p, q, r}\right|\left|x_{p, q, r}\right|$ 
Let us consider a triple sequence defined by

$$
s_{i, j, k}=\left\{\begin{array}{ccccccccc}
1 & 0 & 0 & 0 & 0 & 0 & 0 & . . & . . \\
0 & 0 & 0 & 0 & 0 & 0 & 0 & . . & . . \\
0 & 0 & 0 & 0 & 0 & 0 & 0 & . . & . . \\
. . & . . & . . & . . & . . & . . & . . & . . & . . \\
. . & . . & . . & . . & . & . & . . & . . & . . \\
0 & 0 & 0 & 0 & 0 & 0 & 0 & . . & . . \\
0 & -1 & 0 & 0 & 0 & 0 & 0 & . . & . . \\
0 & 0 & 0 & 0 & 0 & 0 & 0 & . . & . . \\
. . & . . & . . & . . & . . & . . & . . & . . & . . \\
. . & . . & . . & . . & . . & . . & . . & . . & . . \\
0 & 0 & 0 & 0 & 0 & 0 & 0 & . . & . . \\
0 & 0 & 0 & 0 & 0 & 0 & 0 & . . & . . \\
0 & 0 & 1 & 0 & 0 & 0 & 0 & . . & . . \\
. . & . . & . . & . . & . . & . . & . . & . . & . . \\
. . & . . & . . & . . & . . & . . & . . & . . & . . \\
0 & 0 & 0 & 0 & 0 & 0 & 0 & . . & . . \\
0 & 0 & 0 & 0 & 0 & 0 & 0 & . . & . . \\
0 & 0 & 0 & 0 & 0 & 0 & 0 & . . & . . \\
0 & 0 & 0 & \frac{1}{2} & 0 & 0 & 0 & . . & . . \\
. . & . . & . . & . . & . . & . . & . . & . . & . . \\
. . & . . & . . & . . & . . & . . & . . & . . & . . \\
0 & 0 & 0 & 0 & 0 & 0 & 0 & . . & . . \\
0 & 0 & 0 & 0 & 0 & 0 & 0 & . . & . . \\
0 & 0 & 0 & 0 & 0 & 0 & 0 & . . & . . \\
0 & 0 & 0 & 0 & 0 & 0 & 0 & . . & . . \\
0 & 0 & 0 & 0 & -\frac{1}{2} & 0 & 0 & . . & . . \\
. . & . . & . . & . . & . . & . . & . . & . . & . . \\
. . & . . & . . & . . & . & . & . . & . . & . . \\
0 & 0 & 0 & 0 & 0 & 0 & 0 & . . & . . \\
0 & 0 & 0 & 0 & 0 & 0 & 0 & . . & . . \\
0 & 0 & 0 & 0 & 0 & 0 & 0 & . . & . . \\
0 & 0 & 0 & 0 & 0 & 0 & 0 & . . & . . \\
0 & 0 & 0 & 0 & 0 & 0 & 0 & . . & . . \\
0 & 0 & 0 & 0 & 0 & -\frac{1}{2} & 0 & . . & . . \\
. . & . . & . . & . . & . . & . . & . . & . . & . . \\
. . & . . & . . & . . & . . & . . & . . & . . & . . \\
. . & . . & . . & . . & . . & . . & . . & . . & . . \\
& & & & & & & &
\end{array}\right.
$$


Let the triple partial sum $\sum_{i=1, j=1, k=1}^{p, q, r} s_{i, j, k}$ is denoted by $\left(H_{p, q, r}\right)$.

Now we have $\left(H_{p, q, r}\right)$ has the following eight properties

(i) $\left|H_{p, p, p}\right| \leq 1$

(ii) $\left|H_{p, p, p}-H_{p, p, p+1}\right|=0$

(iii) $\left|H_{p, p, p}-H_{p, p+1, p+1}\right|=0$

(iv) $\left|H_{p, p, p}-H_{p, p+1, p}\right|=0$

(v) $\left|H_{p, p, p}-H_{p+1, p+1, p}\right|=0$

(vi) $\left|H_{p, p, p}-H_{p+1, p, p}\right|=0$

(vii) $\left|H_{p, p, p}-H_{p+1, p, p+1}\right|=0$

(viii) $\left|H_{p, p, p}-H_{p+1, p+1, p+1}\right|=\lambda_{p, p, p}$ with $P-\lim _{p, p, p} \lambda_{p, p, p}=0$

By the RH-regularity conditions of $A$ and $B$ we can choose the following index sequences

$\left(l_{t}\right),\left(m_{u}\right),\left(n_{v}\right),\left(\alpha_{l}\right),\left(\bar{\alpha}_{l}\right),\left(\beta_{m}\right),\left(\bar{\beta}_{m}\right),\left(\gamma_{n}\right),\left(\bar{\gamma}_{n}\right)$ and $\left(\delta_{l, m, n}\right)$

Choose $\left(\alpha_{l}\right),\left(\bar{\alpha}_{l}\right),\left(\beta_{m}\right),\left(\bar{\beta}_{m}\right),\left(\gamma_{n}\right),\left(\bar{\gamma}_{n}\right)$ are strictly increasing and $P-\lim _{l, m, n} \delta_{l, m, n}=0$

By the RH-regularity condition $R H_{1}$ gives us

$\Sigma_{p=1, q=1, r=1}^{\alpha_{l}-1, \beta_{m}-1, \gamma_{n}-1}\left|a_{l, m, n, p, q, r}\right|<\delta_{l, m, n}$,

and

$\sum_{p=1, q=1, r=1}^{\alpha_{l}-1, \beta_{m}-1, \gamma_{n}-1}\left|b_{l, m, n, p, q, r}\right|<\delta_{l, m, n}$,

Now $\mathrm{RH}_{3}, \mathrm{RH}_{4}$ and $\mathrm{RH}_{5}$ gives us the following: 


$$
\begin{aligned}
& \Sigma_{p=1, q=\beta_{m}, r=\bar{\gamma}_{n}}^{\alpha_{l}-1, \bar{\beta}_{m}-1, \infty}\left|a_{l, m, n, p, q, r}\right|<\delta_{l, m, n}, \\
& \sum_{p=1, q=\bar{\beta}_{m}, r=\gamma_{n}}^{\alpha_{l}-1, \infty, \bar{\gamma}_{n}-1}\left|a_{l, m, n, p, q, r}\right|<\delta_{l, m, n}, \\
& \sum_{p=\alpha_{l}, q=1, r=\bar{\gamma}_{n}}^{\bar{\alpha}_{l}-1, \beta_{m}-1, \infty}\left|a_{l, m, n, p, q, r}\right|<\delta_{l, m, n}, \\
& \sum_{p=\bar{\alpha}_{l}, q=1, r=\gamma_{n}}^{\infty, \beta_{m}-1, \bar{\gamma}_{n}-1}\left|a_{l, m, n, p, q, r}\right|<\delta_{l, m, n}, \\
& \sum_{p=\bar{\alpha}_{l}, q=\beta_{m}, r=1}^{\infty, \bar{\beta}_{m}-1, \gamma_{n}-1}\left|a_{l, m, n, p, q, r}\right|<\delta_{l, m, n}, \\
& \sum_{p=\alpha_{l}, q=\bar{\beta}_{m}, r=1}^{\bar{\alpha}_{l}-1, \infty, \gamma_{n}-1}\left|a_{l, m, n, p, q, r}\right|<\delta_{l, m, n},
\end{aligned}
$$

and

$$
\begin{aligned}
& \sum_{p=1, q=\beta_{m}, r=\bar{\gamma}_{n}}^{\alpha_{l}-1, \bar{\beta}_{m}-1, \infty}\left|b_{l, m, n, p, q, r}\right|<\delta_{l, m, n}, \\
& \sum_{p=1, q=\bar{\beta}_{m}, r=\gamma_{n}}^{\alpha_{l}-1, \infty, \bar{\gamma}_{n}-1}\left|b_{l, m, n, p, q, r}\right|<\delta_{l, m, n}, \\
& \sum_{p=\alpha_{l}, q=1, r=\bar{\gamma}_{n}}^{\bar{\alpha}_{l}-1, \beta_{m}-1, \infty}\left|b_{l, m, n, p, q, r}\right|<\delta_{l, m, n}, \\
& \sum_{p=\bar{\alpha}_{l}, q=1, r=\gamma_{n}}^{\infty, \beta_{m}-1, \bar{\gamma}_{n}-1}\left|b_{l, m, n, p, q, r}\right|<\delta_{l, m, n}, \\
& \sum_{p=\bar{\alpha}_{l}, q=\beta_{m}, r=1}^{\infty, \bar{\beta}_{m}-1, \gamma_{n}-1}\left|b_{l, m, n, p, q, r}\right|<\delta_{l, m, n}, \\
& \sum_{p=\alpha_{l}, q=\bar{\beta}_{m}, r=1}^{\bar{\alpha}_{l}-1, \infty, \gamma_{n}-1}\left|b_{l, m, n, p, q, r}\right|<\delta_{l, m, n},
\end{aligned}
$$

and $\mathrm{RH}_{6}$ gives us

$$
\Sigma_{p=\bar{\alpha}_{l}-1, q=\bar{\beta}_{m}-1, r=\bar{\gamma}_{n}-1}^{\infty, \infty}\left|a_{l, m, n, p, q, r}\right|<\delta_{l, m, n},
$$

and

$$
\Sigma_{p=\bar{\alpha}_{l}-1, q=\bar{\beta}_{m}-1, r=\bar{\gamma}_{n}-1}^{\infty, \infty, \infty}\left|b_{l, m, n, p, q, r}\right|<\delta_{l, m, n},
$$

We choose the indux sequences $\left(\alpha_{l_{t}}\right),\left(\bar{\alpha}_{l_{t}}\right),\left(\beta_{m_{u}}\right),\left(\bar{\beta}_{m_{u}}\right),\left(\gamma_{n_{v}}\right)$ and $\left(\bar{\gamma}_{n_{v}}\right)$ such that

$$
\alpha_{l_{t}}=\bar{\alpha}_{l_{t-1}}, \beta_{m_{u}}=\bar{\beta}_{m_{u-1}} \text { and } \gamma_{n_{v}}=\bar{\gamma}_{n_{v-1}} \text { for all } t, u, v=1,2,3,4, \ldots \ldots \ldots
$$


We consider the following triple sequence

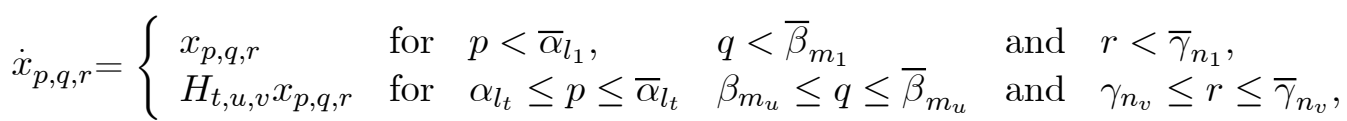

Now for the inequalities $l_{t} \leq l<l_{t+1}, m_{u} \leq m<m_{u+1}$ and $n_{v} \leq n<$ $n_{v+1}$ the above triple sequence $\left(\dot{x}_{p, q, r}\right)$ gives us the following:

$$
\begin{aligned}
& \dot{\chi}_{l, m, n}=\Sigma_{p=1, q=1, r=1}^{\infty, \infty, \infty} a_{l, m, n, p, q, r} \dot{x}_{p, q, r} \\
& =\sum_{p=1, q=1, r=1}^{\alpha_{l}-1, \beta_{m}-1, \gamma_{n}-1} a_{l, m, n, p, q, r} \dot{x}_{p, q, r}+\Sigma_{1 \leq p<\alpha_{l}-1, \beta_{m} \leq q \leq \bar{\beta}_{m}-1}, \\
& \bar{\gamma}_{n} \leq r \leq \infty a_{l, m, n, p, q, r} \dot{x}_{p, q, r} \\
& +\Sigma_{1 \leq p<\alpha_{l}-1, \bar{\beta}_{m} \leq q \leq \infty, \gamma_{n} \leq r \leq \bar{\gamma}_{n}-1} a_{l, m, n, p, q, r} \dot{x}_{p, q, r}+\Sigma_{\alpha_{l} \leq p<\bar{\alpha}_{l}-1,1 \leq q \leq \beta_{m}-1}, \\
& \bar{\gamma}_{n} \leq r \leq \infty a_{l, m, n, p, q, r} \dot{x}_{p, q, r} \\
& +\Sigma_{\bar{\alpha}_{l} \leq p<\infty, 1 \leq q \leq \beta_{m}-1, \gamma_{n} \leq r \leq \bar{\gamma}_{n}-1} a_{l, m, n, p, q, r} \dot{x}_{p, q, r}+\Sigma_{\bar{\alpha}_{l} \leq p<\infty, \beta_{m} \leq q \leq \bar{\beta}_{m}-1}, \\
& 1 \leq r \leq \gamma_{n}-1 a_{l, m, n, p, q, r} \dot{x}_{p, q, r} \\
& +\Sigma_{\alpha_{l} \leq p<\bar{\alpha}_{l}-1, \bar{\beta}_{m} \leq q \leq \infty, 1 \leq r \leq \gamma_{n}-1} a_{l, m, n, p, q, r} x_{p, q, r}+\Sigma_{\bar{\alpha}_{l}-1 \leq p<\infty, \bar{\beta}_{m}-1 \leq q \leq \infty,} \\
& \bar{\gamma}_{n}-1 \leq r \leq \infty a_{l, m, n, p, q, r} \dot{x}_{p, q, r} \\
& +H_{t, u, v} \Sigma_{\alpha_{l} \leq p<\bar{\alpha}_{l}, \beta_{m} \leq q \leq \bar{\beta}_{m}, \gamma_{n} \leq r \leq \bar{\gamma}_{n}} a_{l, m, n, p, q, r} x_{p, q, r} \\
& +\left[H_{t, u, v+1}-H_{t, u, v}\right] \Sigma_{\bar{\alpha}_{l_{t}} \leq p<\bar{\alpha}_{l}, \beta_{m_{u}} \leq q \leq \bar{\beta}_{m_{u}}, \gamma_{n} \leq r \leq \gamma_{n_{v}}} a_{l, m, n, p, q, r} x_{p, q, r} \\
& +\left[H_{t, u+1, v+1}-H_{t, u, v}\right] \Sigma_{\bar{\alpha}_{l_{t}} \leq p<\bar{\alpha}_{l}, \beta_{m} \leq q \leq \beta_{m_{u}}, \bar{\gamma}_{n_{v}} \leq r \leq \bar{\gamma}_{n}} a_{l, m, n, p, q, r} x_{p, q, r} \\
& +\left[H_{t, u+1, v}-H_{t, u, v}\right] \Sigma_{\alpha_{l_{t}} \leq p<\bar{\alpha}_{l_{t}}, \bar{\beta}_{m_{u}} \leq q \leq \bar{\beta}_{m}, \gamma_{n_{v}} \leq r \leq \bar{\gamma}_{n_{v}}} a_{l, m, n, p, q, r} x_{p, q, r} \\
& +\left[H_{t+1, u+1, v}-H_{t, u, v}\right] \Sigma_{\alpha_{l_{t}} \leq p<\bar{\alpha}_{l_{t}}, \beta_{m} \leq q \leq \beta_{m_{u}}, \bar{\gamma}_{n_{v}} \leq r \leq \bar{\gamma}_{n}} a_{l, m, n, p, q, r} x_{p, q, r} \\
& +\left[H_{t+1, u, v}-H_{t, u, v}\right] \Sigma_{\alpha_{l} \leq p<\alpha_{l_{t}}, \bar{\beta}_{m_{u}} \leq q \leq \bar{\beta}_{m}, \gamma_{n_{v}} \leq r \leq \bar{\gamma}_{n_{v}}} a_{l, m, n, p, q, r} x_{p, q, r}
\end{aligned}
$$




$$
\begin{aligned}
& +\left[H_{t+1, u, v+1}-H_{t, u, v}\right] \Sigma_{\alpha_{l} \leq p<\alpha_{l_{t}}, \beta_{m_{u}} \leq q \leq \bar{\beta}_{m_{u}}, \gamma_{n} \leq r \leq \gamma_{n_{v}}} a_{l, m, n, p, q, r} x_{p, q, r} \\
& +\left[H_{t+1, u+1, v+1}-H_{t, u, v}\right] \Sigma_{\bar{\alpha}_{l_{t}} \leq p<\bar{\alpha}_{l}, \bar{\beta}_{m_{u}} \leq q \leq \bar{\beta}_{m}, \bar{\gamma}_{n_{v}} \leq r \leq \bar{\gamma}_{n}} a_{l, m, n, p, q, r} x_{p, q, r}
\end{aligned}
$$

Absolute value properties gives us the following:

$\left|\dot{\chi}_{l, m, n}\right| \leq \Sigma_{p=1, q=1, r=1}^{\alpha_{l}-1, \beta_{m}-1, \gamma_{n}-1}\left|a_{l, m, n, p, q, r}\right|\left|\dot{x}_{p, q, r}\right|+\Sigma_{1 \leq p<\alpha_{l}-1, \beta_{m} \leq q \leq \bar{\beta}_{m}-1}$, $\bar{\gamma}_{n} \leq r \leq \infty\left|a_{l, m, n, p, q, r}\right|\left|\dot{x}_{p, q, r}\right|$

$+\Sigma_{1 \leq p<\alpha_{l}-1, \bar{\beta}_{m} \leq q \leq \infty, \gamma_{n} \leq r \leq \bar{\gamma}_{n}-1}\left|a_{l, m, n, p, q, r}\right|\left|\dot{x}_{p, q, r}\right|+\Sigma_{\alpha_{l} \leq p<\bar{\alpha}_{l}-1,1 \leq q \leq \beta_{m}-1}$, $\bar{\gamma}_{n} \leq r \leq \infty\left|a_{l, m, n, p, q, r}\right|\left|\hat{x}_{p, q, r}\right|$

$+\Sigma_{\bar{\alpha}_{l} \leq p<\infty, 1 \leq q \leq \beta_{m}-1, \gamma_{n} \leq r \leq \bar{\gamma}_{n}-1}\left|a_{l, m, n, p, q, r}\right|\left|\dot{x}_{p, q, r}\right|+\Sigma_{\bar{\alpha}_{l} \leq p<\infty, \beta_{m} \leq q \leq \bar{\beta}_{m}-1,}$, $1 \leq r \leq \gamma_{n}-1\left|a_{l, m, n, p, q, r}\right|\left|\dot{x}_{p, q, r}\right|$

$+\Sigma_{\alpha_{l} \leq p<\bar{\alpha}_{l}-1, \bar{\beta}_{m} \leq q \leq \infty, 1 \leq r \leq \gamma_{n}-1}\left|a_{l, m, n, p, q, r}\right|\left|\dot{x}_{p, q, r}\right|+\Sigma_{\bar{\alpha}_{l}-1 \leq p<\infty, \bar{\beta}_{m}-1 \leq q \leq \infty,}$ $\bar{\gamma}_{n}-1 \leq r \leq \infty\left|a_{l, m, n, p, q, r}\right|\left|\hat{x}_{p, q, r}\right|$

$+\left|H_{t, u, v}\right| \Sigma_{\alpha_{l} \leq p<\bar{\alpha}_{l}, \beta_{m} \leq q \leq \bar{\beta}_{m}, \gamma_{n} \leq r \leq \bar{\gamma}_{n}}\left|a_{l, m, n, p, q, r}\right|\left|x_{p, q, r}\right|$

$+\left|H_{t, u, v+1}-H_{t, u, v}\right| \Sigma_{\bar{\alpha}_{l} \leq p<\bar{\alpha}_{l}, \beta_{m_{u}} \leq q \leq \bar{\beta}_{m_{u}}, \gamma_{n} \leq r \leq \gamma_{n_{v}}}\left|a_{l, m, n, p, q, r}\right|\left|x_{p, q, r}\right|$

$+\left|H_{t, u+1, v+1}-H_{t, u, v}\right| \Sigma_{\bar{\alpha}_{l} \leq p<\bar{\alpha}_{l}, \beta_{m} \leq q \leq \beta_{m_{u}}, \bar{\gamma}_{n_{v}} \leq r \leq \bar{\gamma}_{n}}\left|a_{l, m, n, p, q, r}\right|\left|x_{p, q, r}\right|$

$+\left|H_{t, u+1, v}-H_{t, u, v}\right| \Sigma_{\alpha_{l_{t}} \leq p<\bar{\alpha}_{l_{t}}, \bar{\beta}_{m_{u}} \leq q \leq \bar{\beta}_{m}, \gamma_{n_{v}} \leq r \leq \bar{\gamma}_{n_{v}}}\left|a_{l, m, n, p, q, r}\right|\left|x_{p, q, r}\right|$

$+\left|H_{t+1, u+1, v}-H_{t, u, v}\right| \Sigma_{\alpha_{l_{t}} \leq p<\bar{\alpha}_{l_{t}}, \beta_{m} \leq q \leq \beta_{m_{u}}, \bar{\gamma}_{n_{v}} \leq r \leq \bar{\gamma}_{n}}\left|a_{l, m, n, p, q, r}\right|\left|x_{p, q, r}\right|$

$+\left|H_{t+1, u, v}-H_{t, u, v}\right| \Sigma_{\alpha_{l} \leq p<\alpha_{l_{t}}, \bar{\beta}_{m_{u}} \leq q \leq \bar{\beta}_{m}, \gamma_{n_{v}} \leq r \leq \bar{\gamma}_{n_{v}}}\left|a_{l, m, n, p, q, r}\right|\left|x_{p, q, r}\right|$

$+\left|H_{t+1, u, v+1}-H_{t, u, v}\right| \Sigma_{\alpha_{l} \leq p<\alpha_{l_{t}}, \beta_{m_{u}} \leq q \leq \bar{\beta}_{m_{u}}, \gamma_{n} \leq r \leq \gamma_{n_{v}}}\left|a_{l, m, n, p, q, r}\right|\left|x_{p, q, r}\right|$

$+\left|H_{t+1, u+1, v+1}-H_{t, u, v}\right| \Sigma_{\bar{\alpha}_{l} \leq p<\bar{\alpha}_{l}, \bar{\beta}_{m_{u}} \leq q \leq \bar{\beta}_{m}, \bar{\gamma}_{n v} \leq r \leq \bar{\gamma}_{n}}\left|a_{l, m, n, p, q, r}\right|\left|x_{p, q, r}\right|$

The inequalities in condition (1) give the following:

$\left|\dot{\chi}_{l, m, n}\right| \leq 8 U \delta_{l, m, n}$ 


$$
\begin{aligned}
& +\left|H_{t, u, v}\right| \Sigma_{\alpha_{l} \leq p<\bar{\alpha}_{l}, \beta_{m} \leq q \leq \bar{\beta}_{m}, \gamma_{n} \leq r \leq \bar{\gamma}_{n}}\left|a_{l, m, n, p, q, r}\right|\left|x_{p, q, r}\right| \\
& +\left|H_{t, u, v+1}-H_{t, u, v}\right| \Sigma_{\bar{\alpha}_{l} \leq p<\bar{\alpha}_{l}, \beta_{m_{u}} \leq q \leq \bar{\beta}_{m_{u}}, \gamma_{n} \leq r \leq \gamma_{n_{v}}}\left|a_{l, m, n, p, q, r}\right|\left|x_{p, q, r}\right| \\
& +\left|H_{t, u+1, v+1}-H_{t, u, v}\right| \Sigma_{\bar{\alpha}_{l} \leq p<\bar{\alpha}_{l}, \beta_{m} \leq q \leq \beta_{m_{u}}, \bar{\gamma}_{n_{v}} \leq r \leq \bar{\gamma}_{n}}\left|a_{l, m, n, p, q, r}\right|\left|x_{p, q, r}\right| \\
& +\left|H_{t, u+1, v}-H_{t, u, v}\right| \Sigma_{\alpha_{l_{t}} \leq p<\bar{\alpha}_{l_{t}}, \bar{\beta}_{m_{u}} \leq q \leq \bar{\beta}_{m}, \gamma_{n_{v}} \leq r \leq \bar{\gamma}_{n_{v}}}\left|a_{l, m, n, p, q, r}\right|\left|x_{p, q, r}\right|
\end{aligned}
$$

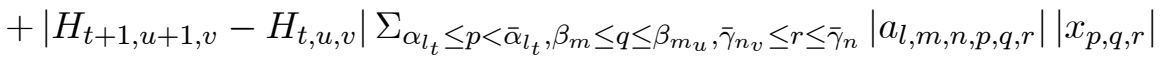

$$
\begin{aligned}
& +\left|H_{t+1, u, v}-H_{t, u, v}\right| \Sigma_{\alpha_{l} \leq p<\alpha_{l_{t}}, \bar{\beta}_{m_{u}} \leq q \leq \bar{\beta}_{m}, \gamma_{n_{v}} \leq r \leq \bar{\gamma}_{n_{v}}}\left|a_{l, m, n, p, q, r}\right|\left|x_{p, q, r}\right| \\
& +\left|H_{t+1, u, v+1}-H_{t, u, v}\right| \Sigma_{\alpha_{l} \leq p<\alpha_{l_{t}}, \beta_{m_{u}} \leq q \leq \bar{\beta}_{m_{u}}, \gamma_{n} \leq r \leq \gamma_{n_{v}}}\left|a_{l, m, n, p, q, r}\right|\left|x_{p, q, r}\right| \\
& +\left|H_{t+1, u+1, v+1}-H_{t, u, v}\right| \Sigma_{\bar{\alpha}_{l} \leq p<\bar{\alpha}_{l}, \bar{\beta}_{m_{u}} \leq q \leq \bar{\beta}_{m}, \bar{\gamma}_{n v} \leq r \leq \bar{\gamma}_{n}}\left|a_{l, m, n, p, q, r}\right|\left|x_{p, q, r}\right|
\end{aligned}
$$

Then we have

$\left|\dot{\chi}_{l, m, n}\right| \leq 8 U \delta_{l, m, n}+\epsilon_{l, m, n}+7 V \lambda_{t, u, v}$

Similarly $\left|\dot{\psi}_{l, m, n}\right| \leq 8 U \delta_{l, m, n}+\epsilon_{l, m, n}+7 V \lambda_{t, u, v}$

Therefore $P-\lim _{l, m, n}(A \dot{x})_{l, m, n}=0$ and $P-\lim _{l, m, n}(A \dot{x})_{l, m, n}=0$ This completes the proof of the theorem.

Theorem 2.2. If $A=\left(a_{l, m, n, p, q, r}\right)$ and $B=\left(b_{l, m, n, p, q, r}\right)$ be two six dimensional $R H$-regular summability matrices sum a bounded triple sequence $\left(x_{p, q, r}\right)$ to the different sums, then there exists a bounded triple sequence which is summed by $A$ but not summed by $B$.

Proof. The proof of the theorem can be established following the technique applied in the above theorem.

Acknowledgement: The author would like to thanks to the University Grants Commission North Eastern Regional Office, Guwahati, India for given the financial support (Ref: No. F.5-330/2015-16/MRP/NERO/1082). 


\section{References}

[1] A. Brudno, Summation of bounded sequences by matrices (in Russian), Recueil Math. (Mat. Sbornik), N.S. 16, pp. 191-247, (1945).

[2] A. Pringsheim, Zurtheorie der zweifachunendlichenzahlenfolgen, Math. Ann. 53, pp. 289-321, (1900).

[3] A. Sahiner, M. Gurdal and K. Duden, Triple sequences and their statistical convergence, Selcuk. J. Appl. Math., 8(2), pp. 49-55, (2007).

[4] A. Sahiner, B. C. Tripathy, Some I-related properties of Triple sequences, Selcuk. J. Appl. Math., 9 (2), pp. 9-18, (2008).

[5] B. C. Tripathy, R. Goswami, Vector valued multiple sequences defined by Orlicz functions, Bol. Soc. Paran. Mat., 33(1), pp. 67-79, (2015).

[6] B. C. Tripathy, R. Goswami, Multiple sequences in probabilistic normed spaces, Afrika Matematika, 26(5-6), pp. 753-760, (2015).

[7] B. C. Tripathy, R. Goswami, Fuzzy real valued p-absolutely summable multiple sequences in probabilistic normed spaces, Afrika Matematika, 26 (7-8), pp. 1281-1289, (2015).

[8] B. C. Tripathy, R. Goswami, On triple difference sequences of real numbers in probabilistic normed spaces, Proyecciones J. Math., 33(2) , pp. 157-174, (2014).

[9] B. C. Das, Some I-convergent triple sequence spaces defined by a sequence of modulus function, Proyecciones J. Math. (Accepted), (2017).

[10] G. M. Robison, Divergent double sequences and series, Trans. Amer. Math. Soc., 28, pp. 50-73, (1926).

[11] H. J. Hamilton, Transformations of multiple sequences, Duke Math. Jour., 2, pp. 29-60, (1936).

[12] L. L. Silverman, On the definition of the sum of a divergent series, $\mathrm{Ph}$. D. Thesis, University of Missouri Studies, Math. Series I, pp. 196, (1913).

[13] O. Toeplitz, Uber allgenmeine linear mittelbrildungen, Prace Mat. Fiz. (Warsaw) 22, (1911). 
[14] R. F. Patterson, Four dimensional characterization of bounded double sequences, Tamkang J. Math, 35(2), pp. 129-134, (2004).

[15] S. Debnath, B. Sharma and B. C. Das, Some Generalized Triple Sequence Spaces of Real Numbers, J. Nonlinear Anal. Opti. 6(1), pp. 71-79, (2015).

[16] S. Debnath and B. C. Das, Some New Type of Difference Triple Sequence Spaces, Palestine J. Math.Vol. 4(2), pp. 284-290, (2015).

[17] S. Debnath, B. C. Das, D. Bhattacharya and J. Debnath, Regular Matrix Transformation on Triple Sequence Spaces, Bol. Soc. Paran. Mat., 35(1), pp. 85-96, (2017) (In Press).

\section{Bimal Chandra Das}

Department of Mathematics

Govt. Degree College,

Kamalpur-799285

(Affiliated to Tripura University)

Dhalai, Tripura,

INDIA

e-mail : bcdas3744@gmail.com 